\title{
¿Qué hay de malo en la prostitución?
}

\author{
PABlo AgUAYO WESTWOOD* \\ Universidad de Chile (Chile) \\ paguayo@derecho.uchile.cl
MARÍA JOSÉ CALDERÓN HERNÁNDEZ**
Universidad de Chile (Chile)
maria.calderon.h@ug.uchile.cl

\begin{abstract}
Resumen
En este trabajo se exploran y evalúan los diferentes argumentos morales que en el debate contemporáneo se han ofrecido a favor y en contra de legalizar la prostitución. En primer lugar, se analizan las posiciones que ven en la regulación la mejor alternativa para enfrentar los problemas que conlleva esta actividad. En segundo lugar, se discuten los argumentos abolicionistas que tienen por objeto erradicar la prostitución bajo el argumento de que esta práctica descansaría sobre una injusticia de género. Al finalizar se discute la necesidad de considerar tanto los argumentos morales como los datos empíricos con el objetivo de determinar la política que menos perjuicios traiga para quienes la ejercen.
\end{abstract}

Palabras clave: prostitución, feminismo, liberalismo, injusticia de género, autorrespeto

\section{What is wrong with prostitution?}

\begin{abstract}
This article assesses the moral arguments presented in the contemporary debate on the legalization of prostitution. The first section analyzes the positions that defend legal regulation as the best alternative to face the problems that this activity entails. The second section discusses abolitionist arguments whose purpose is to support an eradication policy since this practice rests on a gender injustice. Finally, the need to consider both moral arguments and empirical data are discussed for determining the best policy for the treatment of prostitution.
\end{abstract}

Key words: prostitution, feminism, liberalism, gender injustice, self-respect.

\footnotetext{
* Doctor en Ética y Democracia. Profesor Asociado de la Facultad de Derecho de la Universidad de Chile. Es autor de Reconocimiento, justicia y democracia (2018) y "Rawls y las críticas al paradigma liberal distributivo. Una defensa de la justicia como equidad a partir de la idea de autorrespeto" (2017).

** Estudiante de $5^{\circ}$ año de la Licenciatura en Ciencias Jurídicas y Sociales en la Universidad de Chile. Ayudante en los cursos de Filosofía moral y Teorías de la justicia en la Facultad de Derecho de la Universidad de Chile. Ayudante de investigación en proyecto FONDECYT de Iniciación No 11170230.

Este artículo forma parte del proyecto FONDECYT de Iniciación No 11170230: "Bases sociales del autorrespeto como métrica de la justicia social". Agradecemos a los evaluadores de Veritas sus sugerencias y comentarios para mejorar este trabajo.
} 
INTRODUCCIÓN

Desde el feminismo de la primera ola la prostitución ha estado bajo escrutinio moral y político al ser una clara consecuencia y manifestación de las desigualdades propias de una cultura patriarcal (Addams, 1914: 910, 142-144, 204-217). Asimismo, las feministas de la segunda ola examinaron la prostitución como un fenómeno social vinculado a un espectro más amplio de relaciones de dominación y centraron su atención en aquellas instituciones opresivas que finalmente permiten a los hombres controlar a las mujeres. En esta línea de argumentación Pateman escribió en El contrato sexual que el contrato de matrimonio era fundamental para el patriarcado en la medida en que facilitaba una forma socialmente aceptable que los hombres tenían para acceder a los cuerpos de las mujeres. Pateman vio que la prostitución era una extensión de esta forma de opresión, y que la institución de la prostitución ofrecía privilegios a los hombres para comprar los servicios sexuales de las mujeres (1988: 189).

En cuanto fenómeno social la prostitución también ha motivado una diversidad de reflexiones sociológicas, morales y jurídicas. En los últimos años estas han tomado más fuerza debido a la irrupción del movimiento feminista de la tercera ola, así como al desarrollo de la teoría crítica feminista. De hecho, en la actualidad es posible reconocer una gran variedad de posiciones sobre la práctica, el comercio y la legalidad de la prostitución. Estas posiciones se han organizado a partir de las diferentes formas en las que es posible valorar el papel de la mujer prostituida. De este modo, la visión que consideraba a la prostituta como alguien que realiza una actividad criminal que atenta contra la moral y las buenas costumbres ha sido desplazada por nuevas perspectivas, tales como aquellas que conciben a la prostitución como un trabajo similar a cualquiera, y otras que la consideran una práctica que perpetúa la violencia sexual hacia las mujeres ${ }^{1}$.

Para profundizar sobre dicha discusión en este artículo analizaremos los argumentos presentados por dos perspectivas antagónicas, a saber, la que pretende regular y legalizar la prostitución, así como la que tiene por objeto abolirla. Si bien en principio este pareciera un debate entre solo dos

1 Entre quienes consideran que es un trabajo como cualquier otro se encuentran Primoratz (1993), Ericsson (1980) y Nussbaum (1998). Por otra parte, entre quienes la consideran como una práctica que perpetua la violencia hacia las mujeres destacan Shrage (1989 y 1994), Satz (1995) y Raymond (2004). Esta lectura dicotómica entre victimización y explotación en contraste con agencia y elección ha sido criticada por Sander, O'Neill y Pitcher (2018: 1, 14-16). Si bien compartimos sus argumentos, nos parece que de modo introductorio es razonable presentar la discusión marcando estas posiciones más extremas. Como el lector observará más adelante, también nosotros nos distanciaremos de esta lectura binaria. 
formas de abordarla (legalizar versus abolir), en la discusión contemporánea De Marneffe (2009) y Shrage (2016b) han distinguido cuatro posiciones que se traducen en cuatro políticas diferentes con sus respectivos fundamentos morales ${ }^{2}$. Cabe destacar que el particular entendimiento que se tenga de la prostitución determinará tanto el tipo de política que se pretenda llevar adelante, como el tipo de argumento moral que su ofrezca para su defensa. De este modo, la naturaleza de los argumentos estará estrictamente determinada por el hecho de concebir esta actividad como una de carácter laboral, o como una forma de naturalización de la violencia de género.

En la primera parte de este artículo analizaremos aquellas posiciones que ven en la regulación la mejor alternativa para enfrentar los problemas que conlleva la práctica de la prostitución. La tesis central de esta posición es el reconocimiento de una relación contractual en la práctica de la prostitución, relación que debe ser legalizada con miras a promover el bienestar de aquellas mujeres que la ejercen. En la segunda parte revisaremos la postura abolicionista que tiene por objeto erradicar la práctica de la prostitución bajo el argumento de que esta descansaría sobre una injusticia de género. Para esta visión la prostitución encarnaría la legitimación de la violencia sexual hacia las mujeres. Para alcanzar esto último revisaremos no solo las críticas en contra de los argumentos contractualistas que equiparan la práctica de la prostitución a otros trabajos, sino que también valoraremos la fuerza que tienen los argumentos consecuencialistas. Finalmente,

2 Estas políticas son: (i) probibición: se refiere a cualquier conjunto de políticas que penaliza la venta y compra de servicios sexuales, o que prohíbe categóricamente el comportamiento asociado con la venta o compra de dichos servicios; (ii) abolición: se refiere a cualquier conjunto de políticas que ni penaliza la venta de servicios sexuales como tal, ni tampoco aquellas actividades estrechamente relacionadas, como por ejemplo ofrecer servicios en la calle. Esta política sí criminaliza la compra de servicios sexuales, así como el comportamiento que se usa comúnmente para obtener tales servicios. Asimismo, esta política también criminaliza la operación de negocios tales como burdeles o casas de tolerancia; (iii) regulación: se refiere a aquellas políticas que no penalizan ni la venta ni la compra de servicios sexuales como tal, ni tampoco las actividades estrechamente relacionadas. El rasgo distintivo de esta política es que sí impone restricciones a la venta, compra y operación de un negocio sexual, restricciones que no se aplican a otros comercios. Como ejemplo de lo anterior es el aumento de la edad mínima para ejercer el comercio sexual (y tener un contrato) de 18 a 21 años, la zonificación y ciertas normas de salud; (iv) total despenalización: se refiere a cualquier conjunto de políticas en las que el derecho penal trata al comercio sexual como a cualquier otro comercio; aplica las mismas leyes que utiliza para regular otros trabajos y no impone restricciones en la publicidad de servicios sexuales, como tampoco a la operación del comercio sexual. La despenalización completa se conoce como una política de normalización o laissez-faire (De Marneffe, 2009: 29-31). 
discutiremos la necesidad de considerar tanto los argumentos morales como los datos empíricos con el objetivo de determinar la política que menos perjuicios traiga para quienes la ejercen.

\section{LA LEGALIZACIÓN DE LA PROSTITUCIÓN}

En esta sección analizaremos los argumentos con los que se busca justificar la legalización de la prostitución ${ }^{3}$. La base de esta posición está determinada por la creencia de que cuando una mujer intercambia sexo por dinero, las partes de ese contrato toman una decisión libre y autónoma. A su vez, esta posición reconoce que el propio cuerpo, o el sexo, puede considerarse un bien de consumo. En virtud de lo anterior, los y las defensoras de esta posición sostienen que debería existir un reconocimiento legal de la prostitución, reconocimiento que permita que el contrato sexual se lleve a cabo bajo condiciones que aseguren ciertos derechos laborales para quienes ejercen la actividad.

\subsection{Los argumentos contractualistas}

Uno de los principales argumentos para la legalización de la prostitución hace referencia al valor de la libertad y de la autonomía sexual de las mujeres que entregan su consentimiento en el ejercicio de la prostitución. Para las defensoras de esta posición la libertad de las mujeres se vería minada si intentáramos limitar o prohibir esta actividad, tal como lo proponen las posturas prohibicionistas y abolicionistas. Impedir la prostitución no solo limitaría la autonomía sexual de quienes ofrecen sus servicios, sino también de quienes quieran contratarlos, toda vez que no se permitiría que las partes entren en acuerdos sexuales mutuamente ventajosos. De acuerdo con lo anterior, lo que está en juego en la prostitución no sería la alienación o venta permanente de las capacidades sexuales, sino más bien el intercambio de trabajo sexual legítimo por beneficios económicos, tal como se intercambia fuerza de trabajo por remuneraciones en cualquier otra relación laboral (Schwarzenbach, 1991: 105-106). En este mismo sentido, un entendimiento de la prostitución como relación laboral favorece-

\footnotetext{
3 Si bien las cuatro posiciones identificadas en la introducción son formas de enfrentar legalmente la prostitución, la literatura considera que la postura legalista es aquella defendida por quienes favorecen una política de regulación o descriminalización.
} 
ría la autonomía sexual de las trabajadoras en la medida en que les permitiría conservar el derecho a retirarse de sus contratos laborales en cualquier momento ${ }^{4}$.

La anterior defensa de la prostitución como actividad laboral descansa en una posición contractualista ${ }^{5}$. Quienes defienden esta perspectiva argumentan que la oposición que una sociedad adopta con respecto al comercio sexual se basa en nuestras actitudes anticuadas hacia la sexualidad en general. Asimismo, otro punto en el que se apoya el argumento contractual es la consideración de la sexualidad humana como una necesidad cuya satisfacción es tan importante como el alimento o el abrigo.

El objetivo de los y las defensoras de esta posición es el reconocimiento de un contrato entre quien provee los servicios sexuales y quien los solicita, lo que implica la erradicación de cualquier ley que pretenda criminalizar dicho intercambio. En este sentido, cabe destacar que los y las autoras contractualistas abogan por una reforma al estado actual en el que se encuentra el ejercicio de la prostitución. El intercambio de sexo por dinero debería reformarse en miras de la construcción de un modelo de prostitución sana, lo que en primer lugar implicaría dejar de lado nuestro legado cultural puritano. Por ejemplo, según Ericsson, una soundprostitution debería contar con al menos las siguientes características: (i) despenalización de la práctica y, por tanto, entrega de derechos laborales a la prostituta, (ii) eliminación de la prostitución infantil, (iii) libre elección de la actividad, (iv) disponibilidad equitativa para ambos sexos y (v) desarrollo en un ambiente social liberado de estigmas y prejuicios (Ericsson 1980: 366). Bajo esta concepción de una prostitución sana se debería reconocer el derecho de una persona a vender sus servicios sexuales tal y como las personas venden otros tipos de servicios categorizados como trabajos. Lo anterior implicaría la eliminación de las diferencias sustantivas entre la prostitución y otros tipos de actividad laboral en virtud de que dichas diferencias

$4 \quad$ Esta tesis es al menos discutible. En los países europeos donde se ha legalizado la prostitución (regulación o descriminalización) ha aumentado el tráfico de mujeres desde Sudamérica y Europa del este, llegando incluso a un 300\% por ciento. Además, no solo la estabilidad económica de estas mujeres depende de su trabajo, sino que la de los otros miembros de su familia y, en muchas ocasiones, su permiso de residencia permanente. Véase Raymond (2004) y Shrage (2016a).

5 La literatura se refiere a esta posición como (i) liberal contractarianism, por ejemplo, Pateman utiliza esta expresión en "Defending Prostitution: Charges Against Ericsson" (1983: 561) o (ii) contractarian, por ejemplo, Schwarzenbach en "Contractarians and Feminists Debate Prostitution" (1991). Pateman también utiliza la expresión contractarian en "What's Wrong with Prostitution?" cuando sostuvo que: "The contractarian idea of universal sale of property (services) is a vision of unimpeded mutual use or universal prostitution" (1999: 62). 
estarían sustentadas en consideraciones morales hipócritas que adoptamos frente a ella.

Dichas consideraciones serían especialmente relevantes en la medida en que influyen en los daños y peligros a los que se someten las prostitutas. En este sentido, cabe hacer una breve referencia a la objeción paternalista y a la respuesta que ofrecen autores como Ericsson y Primoratz. Por ejemplo, para Joel Feinberg (1971) y Ronald Dworkin (1989; 2011: 361-371), el paternalismo correspondería a la interferencia en la libertad de acción de una persona que solo se encuentra justificada por razones referidas a su bienestar y felicidad, o por los intereses y valores de quien es coaccionado. En este sentido, quienes abogan por la abolición de la prostitución consideran que su rechazo se vincula a sus riesgos inminentes y sustantivos, riesgos que irían en contra del bienestar y los intereses de la prostituta. Por todo lo anterior, se justificaría la intervención estatal -o un paternalismo legal usando la expresión de Feinberg- con el objetivo de establecer un sistema de normas que o bien restrinjan la prostitución, o bien la supriman definitivamente.

En relación con el punto anterior, Primoratz sostuvo que los riesgos específicos a los que se enfrentaría una persona que ejerce la prostitución son cuatro: (i) enfermedades venéreas, (ii) el comportamiento violento, humillante y no placentero de los clientes, (iii) explotación por parte de madames o proxenetas y (iv) el estatus social extremadamente bajo de las prostitutas que las hace objeto de desprecio y las conduce al ostracismo. Estos daños y riesgos a los que estarían sometidas las prostitutas suelen estar asociados a las razones por las cuales la sociedad debería intentar prevenir que ellas se dediquen al comercio sexual. Sin embargo, para los y las autoras que defienden una posición liberal-contractual dichos peligros y daños no son razón suficiente para considerar que la prostitución sea una actividad negativa que deba prohibirse. Entre otras razones, estas autoras y autores sostienen que esos mismos riesgos y peligros son producidos por la actitud negativa que la sociedad tiene sobre la prostitución, como por ejemplo la condena moral que establece que ella constituye un caso de inmoralidad sexual debido a nuestra herencia valorativa.

El punto anterior se vería demostrado por el hecho de que existe un número importante de profesiones en las que los y las trabajadores se enfrentan a peligros inminentes, pero estos peligros son tolerados y, por tanto, sus actividades no consideradas indeseables. Por ejemplo, en aquellos trabajos en que las personas están expuestas a sustancias tóxicas o explosivas las disposiciones que se toman son de protección con respecto a los factores del ambiente laboral que las vuelve riesgosas, y no una actitud paternalista sobre el agente que decide voluntariamente acceder a dicho trabajo. La conclusión a la que llegan tanto Ericsson como Primoratz 
es que una actitud paternalista hacia la prostitución no es más que el reflejo de nuestra moral conservadora, la que termina dañando a quienes llevan a cabo la prostitución. De esta manera, sería claro que dichos peligros no son razones suficientes para prohibirla, sino que deberían ser tomados en consideración justamente como razones para intentar desengañar a la sociedad y eliminar los prejuicios que tiene en contra de la prostitución y, de este modo, cambiar no solo la legislación, sino las condiciones sociales en las cuales esta práctica se sustenta.

\subsection{Sobre el estigma en torno al trabajo sexual}

Una idea similar a la expuesta en el punto anterior es la que esgrime Martha Nussbaum. El argumento que Nussbaum ofrece a favor de la legalización de la prostitución descansa principalmente en la creencia de que esta actividad es similar a cualquier otro trabajo (1998: 710). Junto a lo anterior, Nussbaum enfatiza que la postura que tradicionalmente tenemos de rechazo hacia la prostitución se basa en prejuicios que estigmatizan tanto a las prostitutas, como a la actividad sexual en sí. Nussbaum cuestiona si la venta de servicios sexuales realmente daña a las mujeres y, por tanto, si realmente estamos frente a una práctica nociva o solo frente a una actividad que podría asimilarse a otras prácticas laborales. Si esto último fuera el caso, la situación en la que se encuentran las prostitutas sería fruto de que su trabajo goza de mala fama producto de diferentes convenciones sociales que tenemos en torno a la relación cuerpo-sexualidad. Para Nussbaum todas las personas ganamos dinero por el uso de nuestros cuerpos a través de las diferentes actividades que realizamos. De esto modo, futbolistas, trabajadores de fábricas, abogados, profesoras de filosofía, obtienen beneficios gracias al uso de su cuerpo. Sin embargo, piensa la autora, quienes ganan dinero por medio de servicios sexuales tienden a ser estigmatizadas y juzgadas bajo una perspectiva diferente de quienes utilizan sus cuerpos para trabajar con otros propósitos. Nussbaum argumenta que dicha diferencia no es legítima y que proviene de la estigmatización de dicha actividad. La pregunta entonces es si este estigma está justificado; y si la respuesta es negativa como responde la autora, entonces no tendríamos razones para deslegitimar el trabajo que realizan las trabajadoras sexuales.

Nussbaum argumenta que los problemas asociados con la prostitución los encontramos en muchos otros tipos de empleos y prácticas sociales y, además, estos no son inherentes al trabajo sexual. Dichos problemas suelen depender de las condiciones inestables y típicas en las que se encuentra una prostituta y, junto a lo anterior, provienen del trato diferenciado que reciben fruto de la estigmatización en la que se ven sumidas. 
Para justificar que el problema que tenemos con la prostitución es su estigmatización Nussbaum la compara con otros trabajos que se le asemejan, y con los cuales esta no tendría diferencias sustantivas. De esta forma, la conclusión de Nussbaum se asimila de alguna manera a la de Ericsson y Primoratz, toda vez que las formas de explotación y abuso que sufren las prostitutas son similares a las que sufren otros trabajadores. Para Nussbaum los abusos deben abordarse mediante mecanismos que mejoren la condición de los trabajadores en general, y no mediante políticas discriminatorias en contra de la prostitución en particular.

Bajo la perspectiva anterior, el verdadero problema de la prostitución sería la ausencia de oportunidades laborales y el bajo control sobre las condiciones de empleo que tienen las mujeres pobres. Para estas mujeres la prostitución se convertiría en la única alternativa para desafiar la situación económica en la que se encuentran. Lo anterior sería problemático ya que dicha carencia podría dejarlas bajo el dominio de organizaciones clandestinas de tráfico y trata de personas, cuya existencia descansa en la propia ilegalidad de la prostitución. En este sentido, la propuesta de Nussbaum considera que la lucha feminista debería promover la expansión de las posibilidades laborales a través de la educación, la capacitación en habilidades y la creación de empleos, junto con un reconocimiento de la misma labor sexual, todo lo anterior con el objeto de que sean reconocidos derechos laborales que le permitan llevar adelante dicha práctica de la forma más segura posible.

A partir de lo anterior, quienes defienden esta posición sostienen que es suficientemente claro que no existen fundamentos para concluir que la prostitución deba prohibirse y, por tanto, esta actividad debe considerarse igual de legítima que otras prácticas y relaciones laborales. De todas maneras, y como lo sostuvimos anteriormente, los sectores que abogan por la legalización de la prostitución no están de acuerdo con la manera en que esta se lleva a cabo actualmente. La razón de lo anterior es que esta forma sí perjudica a las mujeres que la realizan, particularmente a aquellas que eligen la prostitución en base a la falta de otras oportunidades laborales. Para los defensores de esta posición la solución a los problemas asociados a la prostitución es su regulación o legalización, y no su abolición.

Es por todo lo anteriormente dicho que las posiciones legalistas defenderán que la mejor solución para tratar los peligros y riesgos que enfrentan las prostitutas es o bien crear ciertas regulaciones que garanticen mejoren sus condiciones laborales, o derechamente descriminalizar su ejercicio. 


\section{LA ABOLICIÓN DE LA PROSTITUCIÓN}

En contraposición con la visión anteriormente analizada existe otra que considera a la prostitución como una práctica nociva. Esta posición sostiene que el comercio sexual en general -y la prostitución en particularno solo descansa, sino que (re)produce la subordinación de las mujeres, constituyendo así una forma de violencia sexual hacia ellas. En términos legislativos, las y los defensores de esta postura pretenden la instauración de un régimen abolicionista cuyo objeto es reducir o erradicar la práctica de la prostitución a través de mecanismos que criminalicen no solo la compra de servicios sexuales, sino también la figura del proxeneta y la administración de burdeles. Cabe recordar que la postura abolicionista no criminaliza a la mujer por considerarla una víctima del comercio sexual.

Ya en El Segundo Sexo Simone de Beauvoir afirmó que desde el punto de vista económico la prostituta y la mujer casada ocupan posiciones simétricas, pero que es en el caso de la prostituta donde se resumen todas las figuras de la esclavitud femenina (1962: 52-53). Esta línea de pensamiento influyó profundamente en una serie de feministas radicales para quienes la crítica a la prostitución, así como a otros mercados sexuales, se volvió un punto de reflexión filosófica sobre el cual era necesario ofrecer argumentos y tomar posición. Por ejemplo, Kathleen Barry -quien concibió a la prostitución como una industria del sexo- sostuvo que esta era la forma más extrema y cristalizada de la explotación sexual para la subordinación de las mujeres (1995: 11).

Los argumentos que surgen desde esta concepción de la práctica de la prostitución se presentan como críticas a la posición contractualista que, como ya hemos dicho, considera a la prostitución como un trabajo cualquiera, a las prostitutas como agentes autónomos de un mercado lícito y a los proxenetas como emprendedores y pequeños empresarios. Asimismo, desde corrientes más radicales, se ha intentado visibilizar el hecho que es el hombre, y no la mujer, quien demanda que su deseo sexual sea satisfecho.

\subsection{El argumento esencialista}

Una de las primeras y fundantes posturas contemporáneas en contra de la práctica de la prostitución corresponde a la desarrollada por Carol Pateman en su libro El Contrato Sexual (1988). Ya en trabajos anteriores Pateman había defendido el argumento según el cual "la prostitución es moralmente indeseable, sin importar cualquier reforma que sobre ella se lleve a cabo, porque es uno de los ejemplos más gráficos de la dominación de los hombres sobre las mujeres" (1983: 561) En este sentido, Pateman 
critica los argumentos contractuales liberales que tienden a asimilar la prostitución a otro tipo de trabajos. Pateman mantiene un interesante debate académico con Lars Ericsson, a quien le responde algunas de las tesis que él defendió en "Charges Against Prostitution: An Attempt at a Philosophical Assessment” (1980).

Para la autora de El Contrato Sexual la comprensión contractual-liberal de la prostitución defendida por Ericsson oculta algunos de sus aspectos fundamentales, principalmente el hecho que la mayor parte de quienes la ejercen son mujeres. Pateman enfatiza que, si bien en el mercado sexual participan hombres y mujeres, son estas últimas las que en su gran mayoría intercambian sexo por dinero. Asimismo, Pateman sostiene que al afirmar que la práctica de la prostitución depende exclusivamente de una cuestión de distribución de cargas y beneficios económicos, Ericsson pasa por alto el hecho de que dicha práctica no solo depende de este tipo de desigualdades, sino también de relaciones desiguales de dominación y subyugación.

Otro punto central de la posición de Pateman es que ella rechaza equiparar la práctica de la prostitución con otros trabajos asalariados típicamente llevados a cabo por mujeres, tales como el secretariado o la enfermería. La principal razón es que el uso de los servicios de una prostituta difiere de otros contratos laborales en la medida en que los varones que contratan a las prostitutas tienen solo interés en su cuerpo, específicamente en el uso sexual del mismo. Al contrario de lo que defienden autores contractualistas cuando intentan equiparar la prostitución a otros trabajos, Pateman asegura que el problema principal es que los servicios que lleva a cabo la prostituta están relacionados de una manera más íntima con su cuerpo en comparación con otros trabajos asalariados.

Para sustentar la tesis anterior, Pateman nos invita a pensar la conexión que existe entre la sexualidad y el sentido de nuestro propio valor. Es ese valor hacia uno mismo el que llevaría a una prostituta a distanciarse de sí misma cuando lleva a cabo el acto sexual con el hombre que pagó por sus servicios. Por tanto, cuando el sexo se convierte en una mercancía, también se vuelve una mercancía su cuerpo y su si mismo, violándose con ello la relación íntima entre la personalidad y la encarnación física de esta ${ }^{6}$. De esta manera, y debido a que la forma en que se involucra nuestra personalidad a través del acto sexual es diferente a como se involucra en otros

\footnotetext{
6 En esta misma línea de argumentación De Marneffe (2009: 13-17) sostuvo que quizás uno de los puntos más complejos en términos psicológicos y morales del ejercicio de la prostitución es el hecho de que las mujeres que la ejercen se ven permanentemente enfrentadas a una situación de falsa identidad, a una continua simulación emocional de la que depende su éxito.
} 
trabajos, no es posible sostener que la prostitución sea un trabajo como cualquier otro. Sobre el punto anterior cabe destacar que como un modo de proteger su yo íntimo, las prostitutas cambian permanente su nombre de servicio. En esta línea de argumentación Carter \& Giobbe sostuvieron que una mujer que ingresa a la prostitución generalmente adquiere un nuevo nombre, cambia su apariencia y crea un pasado ficticio. Ella hace esto "no tanto para protegerse de la policía, sino para reorganizar su personalidad y así satisfacer la demanda del mercado, y también como un intento de guardar algo de sí misma para sí misma" (1999: 45-46)7.

Asimismo, Pateman remarca la diferencia de género que existe entre el comprador y la prostituta, diferencia que expresaría el estatus inferior de las mujeres. Para Pateman el contrato sexual entre hombres y mujeres revelaría que la construcción patriarcal que sustenta la diferencia entre masculinidad y femineidad no es más que la diferencia política entre libertad y sujeción; y que la manera a través de la cual los hombres reafirman dicha masculinidad sobre las mujeres es por medio del acto sexual, o lo que Pateman llama el dominio sexual. De esta manera, el contrato que estaría a la base del intercambio de sexo por dinero implicaría la reafirmación del hombre por sobre la mujer y, en cierto sentido, la reafirmación de su desigualdad económica, política y social.

Finalmente, desde el trabajo de Pateman surgen otras dos críticas al contractualismo-liberal que se mantienen en el centro del análisis feminista sobre la prostitución. En primer lugar, se critica que se trate a la prostitución como un problema de las mujeres que son prostitutas, y no como un problema de los hombres que la demandan. La percepción de la prostitución como un problema de (las) mujeres implica que muy probablemente se acuse que las críticas a la prostitución muestran menosprecio hacia ellas. Asimismo, dicho enfoque implica que el otro participante del contrato de prostitución escape al escrutinio, es decir, se pasa por alto explicar -y justificar- por qué los hombres exigen que las mujeres vendan sus cuerpos como bienes en el mercado. En segundo lugar, se critica la caracterización del impulso sexual masculino como una necesidad que reclama su satisfacción por medio de la mercantilización de la sexualidad femenina. Para Pateman hay una diferencia evidente que Ericsson omite cuando compara el sexo con otras necesidades humanas, a saber, el hecho de que el sexo

Los problemas psicológicos asociados a esta situación fueron tratados por Farley \& Barkan en: "Prostitution, Violence, and Posttraumatic Stress Disorder" (1998). Estudios empíricos que dan soporte a la tesis anterior se pueden encontrar en Chapkis: Live Sex Acts: Women Performing Erotic Labor (1997), Farley: "Bad for the Body, Bad for the Heart': Prostitution Harms Women Even if Legalized or Decriminalized" (2004) y Herman: "Hidden in Plain Sight: Clinical Observations on Prostitution" (2004). 
no es una necesidad cuya satisfacción determina la vida orgánica de los individuos. Junto con lo anterior, que Ericsson equipare a la prostitución con el sexo sin mutua afección amorosa pasa también por alto que en la prostitución ni siquiera hay un deseo mutuo. De hecho, para Pateman la diferencia entre la expresión recíproca del deseo y la obligación unilateral a realizar ciertos actos sexuales con el único beneficio del pago, es comparable a la diferencia entre libertad y la sujeción.

La idea de que la prostitución no es más que un trabajo como cualquier otro es también resistida por Scott Anderson quien considera que la normalización de esta práctica socava la autonomía sexual de las personas (2002: 770-789). A través de la prostitución las mujeres renuncian a su derecho a la autonomía sexual porque sus trabajos las ponen bajo la obligación contractual de tener relaciones sexuales y, por lo tanto, disminuye el control que ellas tienen sobre cuándo y con quién quieren tenerlas.

Frente a la postura de Nussbaum que compara la prostitución con otros trabajos tales como ser masajista, cantante de ópera o profesora de filosofía, Anderson lleva a cabo un ejercicio hipotético a través del cual ilustra las implicaciones de considerar el trabajo sexual como cualquier otro. El autor considera que tratar el comercio sexual como otra forma de trabajo socavaría tres aspectos de la autonomía sexual: (i) se esperaría de nosotros que participemos en actividades sexuales cuando ciertos incentivos están presentes, (ii) tendríamos menos control sobre nuestras vidas sexuales y, finalmente (iii) nuestra autonomía sexual se vería socavada a través del mercado agresivo de los servicios sexuales, los que alterarían el marco de ideas en relación con el sexo permisible ${ }^{8}$.

Aunque la prohibición (o abolición) de la prostitución no es en sí misma suficiente para garantizar o restaurar la autonomía sexual de aquellas mujeres que la han perdido, el autor considera que la prohibición puede jugar un rol importante en el rechazo a aquellas transacciones de sexo por dinero porque van en la disminución de la autonomía de las mujeres. La prohibición (o abolición) de la prostitución constituye una restricción al tipo de presiones o circunstancias que una sociedad debe permitir que una persona soporte en relación con sus elecciones laborales sexuales. Visto desde esta perspectiva, el debate sobre las diferentes políticas y legislaciones que se deben adoptar frente a la práctica de la prostitución

8 Por otra parte, nuestro empleador podría consignar ciertas tareas sexuales como parte de nuestro contrato. Por otra parte, todas aquellas personas que se encuentran desempleadas, y que cuentan con un seguro social bajo la condición de estar buscando empleo, deberían estar disponibles para trabajar como prostitutas o prostitutos. Todo lo anterior bajo el supuesto de considerar a la prostitución como un trabajo cualquiera. 
debería ir mucho más allá del examen de la voluntariedad de quienes intercambian sexo por dinero, o de la igualdad entre lo que se da y lo que se recibe. El debate debería centrarse por sobre todo en las condiciones de justicia que anteceden y rodean la transacción y cómo estas condiciones asimétricas, tanto económicas como de estatus, son la base del comercio sexual. Como sostuvo Scott Anderson, el problema de la prostitución "debería ser entendido como un asunto de justicia social" (2002: 780).

Tanto para Anderson como para Pateman la degradación que sufrirían las prostitutas estaría unida a cuestiones inherentes a la práctica ${ }^{9}$. En el caso de Pateman estas estarían vinculadas a la íntima relación que existe entre la prostitución y el yo de la mujer, mientras que para Anderson la actividad por sí misma minaría la autonomía sexual de las mujeres. Dado lo anterior, las reformas que se podrían llevar a cabo para cambiar las circunstancias en las que se ejerce la prostitución no impedirían la degradación sufrida por las prostitutas, principalmente porque a pesar de dichos cambios permanecerían necesariamente sus características inherentes.

\subsection{El argumento de la disminución de los daños}

En Liberalism and Prostitution Peter de Marneffe se centra en un tipo específico de prostitución que resulta dañino para las mujeres que lo ejercen, a saber, aquel en el que quienes proveen servicios sexuales lo hacen diariamente a un número de hombres que no conocen (2009: 13-14). El autor está interesado en las consecuencias que dicha forma de prostitución implica para quienes la llevan a cabo. De Marneffe considera que los daños que padecen las prostitutas no son solo físicos, sino también psicológicos; daños que afectan principalmente su autopercepción y autorrespeto. Si nos tomamos en serio estos daños, debería hacernos sentido la existencia de leyes paternalistas que tengan como objetivo disminuirlos mediante la reducción del número de personas que llevan a cabo el trabajo sexual. En

$9 \quad$ Otro punto que resulta importante destacar aquí es la diferencia entre (i) el acto particular de intercambiar sexo por dinero y (ii) la práctica de la prostitución y el comercio que la permite y acompaña. Lo anterior nos lleva a la distinción que Rawls realizó entre justificar una práctica y justificar un caso que cae bajo dicha práctica. En principio parecería que los defensores de la regulación y descriminalización de la prostitución entenderían esta actividad como un caso completamente aislado e independiente de las condiciones sociales que la permiten y fomentan. Solo desde ese punto de vista podría intentar justificarse que la prostitución es un trabajo como cualquiera. En cambio, consideramos que lo que las y los autores abolicionistas están tratando de mostrar es que lo anterior es imposible debido a que no podemos (ni debemos) separara este acto-contrato particular de las condiciones, reglas y prácticas sociales que le dan sustento. Los diferentes argumentos filosóficos necesarios para justificar y distinguir los casos de las reglas se encuentran en John Rawls, “Two Concepts of Rules” (1955: 18-29). 
este sentido, la respuesta frente a legalizar o abolir la prostitución no descansa en una particular preconcepción que tengamos del trabajo sexual (posición esencialista $^{10}$ ), sino más bien en el reconocimiento de los daños que esta produce en las mujeres. Es por lo anterior que la elección de una adecuada política dependerá de si esta logra reducir dichos daños. Para De Marneffe la respuesta deberá finalmente descansar en un equilibrio reflexivo entre nuestras intuiciones sobre lo que es prostitución y lo que la experiencia nos muestre. Al no ser un argumento deductivo, no habrá una respuesta única y esta dependerá tanto del contexto como de las experiencias a las que nos enfrentemos.

Como ya hemos sostenido, el daño que sufren las prostitutas no es negado por quienes pretenden legalizar esta práctica. Tanto Ericsson como Nussbaum están de acuerdo en que actualmente las mujeres que la llevan a cabo resultan dañadas de una u otra forma. Sin embargo, dichos autores atribuyen el daño que sufren las prostitutas tanto a la estigmatización como a los prejuicios que como sociedad tenemos sobre ellas. A diferencia de dichos autores, De Marneffe considera que la manera de disminuir estos daños sería mediante un régimen de abolición permisivo, y no mediante la descriminalización o prohibición. Esta es una conclusión a la cual el autor llega mediante un análisis de los datos empíricos disponibles sobre los daños sufridos por las prostitutas, datos que ofrecen los elementos necesarios para pensar en cuál es la mejor manera de reducir$\operatorname{los}^{11}$.

Para De Marneffe el tipo de prostitución antes descrito resulta especialmente estresante para las mujeres que lo ejercen en la medida en que lo experimentan como humillante y violento, trayendo además la aparición

10 Una aproximación esencialista manifiesta que la venta de trabajo sexual es mala en términos morales porque ella es inherentemente alienante o dañina para quien la ejerce. Quienes defienden esta postura sostienen que el sexo tiene una propiedad intrínseca en virtud de la cual resulta perniciosa su transformación en mercancía, o peor aún, es una clara agresión en contra de la dignidad personal. Para este punto véase Anderson (1993: 45) y Satz (1995: 64).

11 Los datos que para De Marneffe resultan sumamente relevante son aquellos relacionados con la edad promedio que tienen las mujeres al momento de ingresar a la prostitución, las condiciones sociales y económicas que funcionan como marco de dicha decisión, así como el trafico de mujeres, jóvenes y niñas desde países empobrecidos hacia aquellos en los que la prostitución es legal; pero por sobre todo lo más relevante para De Marneffe es la disminución o aumento del número de mujeres, jóvenes y niñas asociado a las diferentes políticas que varios países europeos han tomado. 
de sentimientos de vergüenza y odio hacia sí mismas ${ }^{12}$. La razón de lo anterior no sería ni los prejuiciosos ni nuestra hipocresía, como han sostenido otras autoras, sino más bien el constante riesgo de violencia y abuso hacia las prostitutas. Asimismo, este tipo de prácticas demanda una constante falsedad emocional de su parte ya que permanentemente deben fingir satisfacción y disfrute cuando llevan a cabo el acto sexual, incluso si están siendo maltratadas física y psicológicamente. Todas estas condiciones, asociadas a las del cambio de nombre y renuncia de su identidad mientras están trabajando, producen trastornos de la personalidad que harían muy difícil pensar que es un trabajo similar a cualquier otro. Para De Marneffe este tipo de prostitución exige un agotador tipo de simulación emocional, por lo que "una trabajadora sexual exitosa generalmente debe fingir disfrutar de la compañía de sus clientes y estar sexualmente interesada y excitada por ellos incluso cuando la consideran estúpida, ofensiva, patética o repulsiva" (2009: 13). Como resultado de lo anterior, quienes están en este trabajo llegan a asociar el sexo con la simulación y la manipulación emocional, así como con la explotación y el abuso. Todo lo anterior hace muy difícil que una mujer que ejerce la prostitución pueda disfrutar plenamente de su vida sexual.

De Marneffe sostiene que a la luz de consideraciones paternalistas estaríamos justificados en apoyar una política abolicionista permisiva o una forma de regulación no permisiva. Una política abolicionista permisiva no criminaliza la venta ni el consumo como tal, pero sí las actividades asociadas tales como la operación de burdeles, la solicitud de servicios en la calle, entre otras. Se ha demostrado que una política abolicionista baja no solo las tasas de prostitución, sino que el tráfico de personas para ejercer el comercio sexual, e incluso baja las tasas de tráfico de mujeres menores de

12 Un defensor de una política de descriminalización podría argumentar que las mujeres que ejercen la prostitución asumen deliberadamente ese riesgo. Al respecto cabe destacar que siguiendo a Feinberg (1971: 108-10) es posible distinguir al menos entre actos cuya realización implica una perfecta voluntariedad por parte de los agentes, de aquellos en los que hay completa involuntariedad. Llevando esta distinción a la práctica de la prostitución, podríamos pensar que las así llamadas call girls caerían dentro de la primera clasificación, mientras que una mujer que ha sido traficada para ejercer la prostitución o una menor de edad enganchada a las drogas y bajo el control de un proxeneta caería en la segunda. Si bien esta clasificación puede ser correcta, el gran número de mujeres que ejerce la prostitución se encuentra en una situación más bien intermedia. Como se verá más adelante, al promover una política de descriminalización se está considerando que todas las mujeres que la ejercen lo hacen con perfecta voluntariedad, lo que es no solo dogmática, sino empíricamente discutible. Para una descripción de los diferentes perfiles de mujeres que ejercen la prostitución, véase la sección Who is a prostitute? en Satz (1995: 6667). 
edad (Raymond, 2004) ${ }^{13}$. Por lo anterior se justificaría dicha política en miras de la reducción de daños. En relación con la regulación no-permisiva, esta impone restricciones de edad de consentimiento y de empleo legal más exigentes de lo común. Frente a ambas, el autor se inclina a preferir la versión permisiva del abolicionismo, ya que la segunda resulta poco factible o realizable en la medida en que habría una tendencia natural a pensar que una persona ha alcanzado cierta edad cuando en realidad no lo hecho, sumado a la facilidad que existe para falsificarla

Con respecto a las restantes alternativas, para el autor de Liberalism and Prostitution la prohibición de la prostitución no minimizaría los riesgos y daños que sufren las mujeres, sino más bien minimiza la autonomía de las mujeres y las criminalizaría generando un ambiente de trabajo menos seguro. En la vereda contraria, una política de completa descriminalización -es decir, una política que le entregue el mismo trato que a otras actividades comerciales- permitiría que más niñas y mujeres vendan sus servicios sexuales, lo cual aumentaría la cantidad de mujeres expuestas al daño.

Cabe destacar que la tesis defendida por Peter de Marneffe no descansa en lo absoluto en criterios moralistas, tales como que la promiscuidad es un vicio o que el intercambio de sexo por dinero es intrínsecamente malo, sino más bien en el claro hecho de que ciertas prácticas y actividades son dañinas para quienes las ejercen, o que aquellas personas que están vinculadas a ellas pueden llegar a estar en una peor situación como resultado de su ejercicio (De Marneffe, 2009: 55). Dado lo anterior, el autor no

13 Por ejemplo, en los países donde se ha legalizado la prostitución, no se han percibido los beneficios prometidos ni se han satisfecho las expectativas de los argumentos liberales-contractuales. En los Holanda las mujeres dedicadas al comercio sexual señalan que la legalización o la despenalización de la industria del sexo no borra el estigma de la prostitución. Dado que las mujeres deben registrarse, ellas pierden su anonimato y por tanto son más vulnerables a ser estigmatizados como "putas", y esta identidad las sigue a todas partes. Asimismo, se vería un aumento de la prostitución infantil. La organización ChildRight con sede en Ámsterdam estima que el número de niños en prostitución ha aumentado en más del 300\% entre 1996-2001, pasando de 4.000 niños en 1996 a 15.000 en 2001 (Bindel \& Kelly, 2003: 15). ChildRight estima que al menos 5.000 de estos niños en la prostitución holandesa se trafican desde otros países, siendo buena parte niñas nigerianas. Finalmente, la legalización/descriminalización de la prostitución aumenta su demanda. Con la despenalización de la industria del sexo muchos hombres que anteriormente no se habrían arriesgado a pagar para tener sexo, ahora consideran que la prostitución es aceptable. La legalización de la prostitución enviaría el mensaje a las nuevas generaciones de hombres y niños que las mujeres son productos sexuales y que la prostitución es diversión inofensiva. Frente a dichos datos cabe destacar que la experiencia de Suecia es la más exitosa en términos de datos empíricos. Una política abolicionista como la sueca ha demostrado que bajan no solo las tasas de prostitución, sino que el tráfico de personas para ejercer el comercio sexual e incluso baja las tasas de tráfico de menores de edad (Swedish Institute, 2010). 
critica la práctica de la prostitución por ser mala (wrong) o inmoral, sino más bien porque es una actividad imprudente y desaconsejable.

\subsection{El argumento de la contingencia}

Otro grupo de teóricos y teóricas consideran que la degradación que sufren las prostitutas se debe a ciertas características contingentes presentes en su ejercicio, siendo la permanencia de dichos rasgos los que hacen que dicha actividad sea inaceptable.

En el marco de la discusión sobre los límites morales del mercado Debra Satz considera que algunos de estos deberían suprimirse cuando tienen una o más de las siguientes características: (i) producen resultados extremadamente perjudiciales para los participantes del intercambio o bien para terceros, (ii) resultan extremadamente perjudiciales para la sociedad (iii) en ellos se da un conocimiento y capacidad de acción muy débil o altamente asimétrico por parte de uno -o de un grupo- de sus participantes y (iv) reflejan -y exacerban- las extremas vulnerabilidades subyacentes a una de las partes de la transacción (Satz, 2010: 95-99). Es dentro de dicho marco que la autora cuestiona la validez de los mercados de servicios sexuales al considerar que estos cuentan con varias de las características antes mencionadas. Si bien Satz considera que dichas características no son contingentes, al persistir otorgarían a los estados legitimidad para prohibirlos o restringirlos.

Satz defiende lo que ella llama la tesis de asimetría. Esta tesis sostiene que existe una asimetría entre la prostitución y otras formas de trabajo que permitiría afirmar que la venta de actividades sexuales y reproductivas es inapropiada. Satz critica a quienes consideran que la asimetría es fundamentalmente económica o esencial y propone considerar un enfoque igualitario para tratar los problemas asociados a esta actividad ${ }^{14}$. Para Satz lo que está mal (wrongness) con la prostitución es su estrecha relación con la inequidad de género, relación que se sustenta en dos dimensiones: una primera de carácter económico que involucra disparidad del ingreso, la segregación en el trabajo y la división inequitativa del trabajo reproductivo en el hogar; y una segunda inequidad de estatus que involucra los estereotipos negativos hacia las mujeres, la distribución desigual del poder, la marginalización y el estigma. Reconocidas estas dos formas de desigualdad la autora defiende que ambas se entrelazan al intentar responder qué está mal

\footnotetext{
14 Desde la aproximación económica se considera que lo malo y dañino de la prostitución descansa en que sus consecuencias son ineficientes ya que generan externalidades negativas tales como enfermedades, culpa y costos para terceros (para la familia, por ejemplo).
} 
con la prostitución. En este sentido, la decisión de las mujeres de entrar en el comercio sexual debe ser vista no solo atendiendo a sus desiguales oportunidades sociales, sino también a sus menores ingreso. La autora sugiere que esta actividad representa y construye y una imagen desigual de género al establecer una clase de mujeres como inferior, una clase que es vista como sirvientas sexuales de los hombres. En este sentido, la prostitución tal y como la conocemos no es separable de la cultura que marginaliza, construye estereotipos y estigmatiza a las mujeres. Para Satz el mercado del trabajo sexual femenino descansa y refuerza estos patrones de discriminación.

Otro argumento centrado en los rasgos contingentes presentes en la prostitución es el que desarrolla Laurie Shrage. La autora considera que la comprensión de los problemas que aquejan a las prostitutas debe enfocarse desde ciertos principios culturales que sustentan su actual práctica. Para Shrage la prostitución, tal y como es concebida y ejercida en nuestra sociedad, es opresiva hacia las mujeres principalmente porque su ejercicio depende de la aceptación de ciertos principios cuya formulación sirven para marginarlas términos sociales y políticos. En este sentido, es el contexto cultural en el cual la prostitución opera el que termina por perpetuar valores y creencias patriarcales perniciosas para las mujeres que la ejercen. Para la autora, existen cuatro principios que sustentan el significado social que actualmente tenemos sobre la prostitución, a saber, (i) la posesión universal un potente impulso sexual, (ii) el dominio natural de los hombres, (iii) el sexo contamina a las mujeres y (iv) la reificación de la practica sexual (Shrage, 1989: 352-358). Todas estas convicciones culturales se encontrarían presentes en nuestra sociedad y estructurarían el significado que la prostitución tiene para nosotros. El argumento es contingente toda vez que, si no existieran dichas concepciones culturales sobre los hombres y mujeres y sus relaciones entre ellos, la práctica de la prostitución podría sustentarse sobre otras convicciones culturales que no necesariamente serían dañinas para las mujeres. En este sentido, para Shrage la prostitución no es una aberración social, sino más bien una consecuencia de ciertos valores que conforman nuestras instituciones y prácticas sociales. De esta manera, Shrage considera que las feministas deberían oponerse a la existencia de la prostitución tal como se ejerce hoy en día dado que los principios que sustentan y organizan la industria sexual en la que su trabajo se ampara descansan en asimetrías de género perniciosas en muchos dominios de nuestra vida social. Tolerar la prostitución implicaría tolerar un trato opresivo hacia las mujeres. 


\section{CONCLUSIONES}

Como hemos visto en este artículo, la práctica de la prostitución he generado un interesante debate acerca de cuál es la mejor política para tratar con los efectos negativos que produce en las mujeres que la ejercen. Esta discusión ha permeado el debate académico feminista llevando a las principales autoras a un desacuerdo que parece irreconciliable. Asimismo, varios autores y autoras desde perspectivas no feministas han intentado aportar a la discusión ofreciendo argumentos para defender alguna de las cuatro políticas que constituyen el marco de discusión, a saber, prohibir, abolir, regular o descriminalizar.

En la primera parte exploramos los principales argumentos a favor de regularizar o descriminalizar la prostitución. El primero de ellos fue el argumento liberal-contractual defendido por Igor Primoratz y Lars Ericsson quienes consideran que, si dos adultos de manera voluntaria consienten un acuerdo económico respecto a una determinada actividad sexual, parece completamente absurdo mantener que hay algo intrínsecamente incorrecto en ello. Para estos autores si relación entre las prostitutas y su cliente estuviese mediada por un contrato, entonces ellas podrían disfrutar de una mejor protección sanitaria, policial y legal, lo que les permitiría a su vez un control más efectivo en contra del abuso y el comportamiento agresivo de los clientes. En esta primera parte también examinamos los argumentos que Martha Nussbaum ofreció para legalizar la prostitución, que, si bien son de carácter liberal, están más bien centrados en cómo nuestro rechazo hacia la prostitución se basa en el estigma que como sociedad tenemos hacia las mujeres que la ejercen.

En la segunda parte examinamos los principales argumentos que se oponen a la normalización del mercado sexual. Analizamos en primer lugar cómo desde la perspectiva esencialista de Pateman y Anderson se justificaría una política abolicionista no-permisiva por el hecho de que la prostitución sería uno de los ejemplos más evidentes de la dominación del hombre por sobre la mujer. En segundo lugar, revisamos la posibilidad de defender una posición abolicionista permisiva a partir de la consideración otros factores, principalmente de los daños que produce la prostitución en las mujeres. En este orden de ideas podemos citar a Peter de Marneffe quien defiende un argumento paternalista según el cual, al ser la prostitución dañina para un número muy importante de mujeres que la ejercen, deberíamos generar leyes que lo reduzcan. Para De Marneffe la mejor alternativa sería una política abolicionista, no solo porque los datos empíricos muestran que esta disminuiría las tasas de prostitución, sino que también porque una política como esta disminuiría el tráfico de personas para ejercer el comercio sexual. 
Al finalizar la segunda parte vimos hasta qué punto las razones para oponerse a la prostitución pueden basarse en el hecho de que existen ciertas características que, si bien no le son inherentes, podrían de igual modo degradar a las mujeres que la ejercen. Asimismo, la revisión de la tesis de Debra Satz sobre los mercados nocivos en general, y de la prostitución en particular, nos permitió ver cómo este tipo de mercados genera una importante contribución a valorar de manera inferior el estatus social de la mujer. Para Satz la prostitución es nociva no solo por los efectos que produce en la percepción que los hombres tienen de las mujeres como siervas sexuales, sino también por los efectos emocionales devastadores que produce en la propia percepción que las mujeres tienen de sí. Todo lo anterior, sostuvo Satz, evidencia que la prostitución es incorrecta en la medida en que contribuye a perpetuar una forma dominante de desigualdad de estatus entre hombres y mujeres. En la misma línea de argumentación, vimos que Laurie Shrage plantea la necesidad de considerar el contexto cultural al momento de evaluar las prácticas sexuales y sus específicas connotaciones. Atendiendo a este enfoque contextual, la autora sostuvo que la industria del sexo se estructura a partir de una serie de actitudes y valores que son opresivos hacia las mujeres.

Frente a todo lo anterior, nos parece sumamente necesario que al momento de evaluar cualquier modificación a la regulación del trabajo sexual deben considerarse los debates académicos contemporáneos sobre esta materia, así como los datos más efectivos y actualizados. Como sugirió De Marneffe, cualquier política que quiera mejorar las condiciones de las mujeres que ejercen la prostitución debe poner en equilibrio nuestras intuiciones sobre esta actividad con los datos de la experiencia. De no hacerlo, podríamos llegar a considerar equivocadamente que la prostitución se reduce a una mera relación contractual aislada de todo un contexto social en el cual dicha práctica se lleva a cabo. Legislar en base a prejuicios, o a información incompleta, podría ser aún más perjudicial para las mujeres que intercambian sexo por dinero.

\section{REFERENCIAS}

Anderson, E. (1993). V alue in etbics and economics. Cambridge, Mass; London: Harvard University Press.

Anderson, S. (2002). Prostitution and Sexual Autonomy: Making Sense of the Prohibition of Prostitution. Ethics, 112(4), 748-780.

Addams, J. (1914). A new conscience and an ancient evil. New York: Macmillan.

Barry, K. (1995). The prostitution of sexuality. New York: New York University Press.

Beauvoir, S. (1962). El Segundo Sexo. Buenos Aires: Siglo Veinte. 
Bindel, J. \& Kelly, L. (2003). A Critical Examination of Responses to Prostitution in Four Countries: Victoria, Australia; Ireland; the Netherlands; and Sweden. London: London Metropolitan University.

Carter, V. \& Giobbe, E. (1999). Duet: Prostitution, Racism and Feminist Discourse. Hastings Women's Law Journal, 10(1), 37-57.

Chapkis, W. (1997). Live sex acts: Women performing erotic labor. London: Cassell.

De Marneffe, P. (2009). Liberalism and Prostitution. Oxford: Oxford University Press.

Dworkin, R. (1989). Liberal Community. California Law Review, 77(3), 49-74.

Dworkin, R. (2011). Justice for Hedgehogs. Cambridge, Mass.: Belknap Press of Harvard University Press.

Ericsson, L. (1980). Charges Against Prostitution: An Attempt at a Philosophical Assessment. Ethics, 90(3), 335-366.

Farley, M. (2004). "Bad for the Body, Bad for the Heart": Prostitution Harms Women Even If Legalized or Decriminalized. Violence Against Women, (10), 1087-125.

Farley, M., \& Barkan, H. (1998). Prostitution, Violence, and Posttraumatic Stress Disorder. Women \& Health, 27(3), 37-49.

Feinberg, J. (1971). Legal Paternalism. Canadian Journal of Philosophy, 1(1),105-124.

Herman, J. (2004). Hidden in Plain Sight: Clinical Observations on Prostitution. Journal of Trauma Practice, 2(3-4), 1-13.

Nussbaum, M. (1998). "Whether from Reason or Prejudice": Taking Money for Bodily Services. Journal of Legal Studies, 27(2), 693-724.

Pateman, C. (1983). Defending Prostitution: Charges Against Ericsson. Ethics, 93(3), 561-565.

Pateman, C. (1988). The Sexual Contract. Cambridge: Polity Press.

Pateman, C. (1999). What's Wrong with Prostitution? Women's Studies Quarterly, 27(1-2), 53-64.

Primoratz, I. (1993). What's Wrong with Prostitution? Philosophy, 68(264), 159182.

Rawls, J. (1955). Two Concepts of Rules. The Philosophical Review, 64(1), 3-32.

Raymond, J. (2004). Ten Reasons for Not Legalizing Prostitution and a Legal

Response to the Demand for Prostitution. Journal of Trauma Practice, 2(3-4), 315-332.

Sander, T., O’Neill M. \& Pitcher, J. (2018) Prostitution: sex work, policy and politics. London: SAGE.

Satz, D. (1995). Markets in Women's Sexual Labor. Ethics, 106(1), 63-85.

Satz, D. (2010). Why Some Things Should Not Be for Sale: The Moral Limits of Markets.

Oxford: Oxford University Press.

Schwarzenbach, S. (1991). Contractarians and Feminists Debate Prostitution.

New York University Review of Law and Social Change, 18(1), 103-130.

Shrage, L. (1989). Should Feminists Oppose Prostitution. Ethics, 99(2), 347-361.

Shrage, L. (1994). Moral dilemmas of feminism: Prostitution, adultery, and abortion. New York; London: Routledge. 
Shrage, L. (2016a). African Americans, HIV, and mass incarceration. The Lancet, 388(10049), E2-E3.

Shrage, L. (2016b). Feminist perspectives on sex markets. Stanford encyclopedia of philosophy. https://plato.stanford.edu/archives/fall2016/entries/feministsex-markets/

Swedish Institute. (2010). Selected extracts of the Swedish government report SOU 2010:49: "The Ban Against the Purchase of Sexual Services. An evaluation 1999-2008”. https://ec.europa.eu/anti-trafficking/publications/ban-against-purchase-sexual-services-evaluation-1999-2008_en 